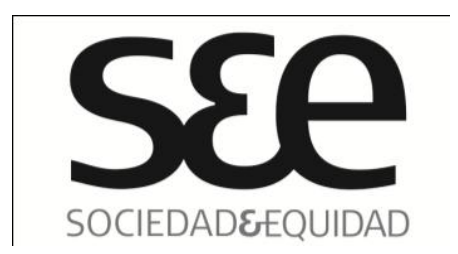

\section{Movimientos sociales y género La siembra feminista de La Vía Campesina}

Social movements and gender: the feminist sow of The Via Campesina

Nombre:

Filiación:

País:

Correo:

\author{
Fernanda Palacios Sepúlveda (1) \\ Investigadora Independiente \\ Chile \\ palaciosfda@gmail.com
}

\title{
RESUMEN
}

Buena parte de los movimientos sociales que han tenido como horizonte la creación de un nuevo "modelo de desarrollo" han considerado, básicamente, la dimensión económico y política como eje análisis, relegando a un segundo plano su dimensión cultural, y dentro ella invisibilizando la variable de género como eje estructurante de la desigualdad social. Por ello, esta investigación se planteó como objetivo, analizar cómo se ha ido integrando la perspectiva de género en un movimiento concreto que ha reconocido la importancia de integrarla como eje del mismo; siendo este el caso de La Vía Campesina. Se utilizó como metodología el análisis de marcos, el cual permite identificar cómo el movimiento se construye a sí mismo, y cómo configuran tanto el diagnóstico como la solución de su problema. Las principales conclusiones apuntan a que, por un lado, hay una apuesta, básicamente de las secciones femeninas, por posicionar la dimensión de género como un eje estructurante, y por otro, que ha existido cierta permeabilidad del movimiento a esa demanda. También se evidencia la emergencia de una consciencia feminista, más cercana a los llamados "feminismos del tercer mundo" que del occidental hegemónico, que podría, eventualmente, llegar a impregnar al movimiento en su conjunto.

\section{PALABRAS CLAVES}

Movimientos sociales, mujeres, género, Latinoamérica, análisis de marcos.

\footnotetext{
${ }^{1}$ La autora es socióloga de la Universidad de Chile. Máster en Estudios Feministas por la Universidad Complutense de Madrid y Magíster en Investigación Participativa para el Desarrollo local por la misma casa de estudios. Sus líneas de investigación son: movimientos sociales, teoría y política feminista, y metodologías y democracias participativas.
} 


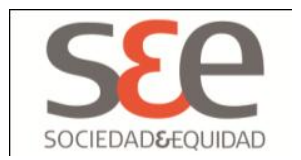

\begin{abstract}
Many of the social movements that have made efforts to create a "developement model", have primarily considered the economical and politic dimension for analysis, relegating the cultural dimension as a less important component, invisibilizing gender issues as a central axis of social inequality. Therefore, this research analyzes how a gender perspective has been integrated in a specific social movement recognizing its importance, "La Vía Campesina". The methodology used is a frame analysis, because it allows identifying how the movement constructs itself, and how they formulate the diagnosis and the solutions of their problems. The main conclusions are, on one hand, that the women's sections position the gender dimension as a central axis and on the other hand, that the movement has shown certain permeability to this demand. It also points to the emergency of a feminist consciousness, closer to the so-called "third world feminism" than to the hegemonic western feminism, which could eventually come to permeate the movement as a whole.
\end{abstract}

\title{
KEYWORDS
}

Social movements, women, gender, Latin America, frame analysis.

\section{INTRODUCCIÓN}

En América Latina, principalmente entre la década de los ' 70 y ' 80 , se produjo una gran cantidad de estudios e investigaciones empíricas sobre la participación de las mujeres en organizaciones de base y movimientos populares referidos a la extensión de derechos sociales, y en caso de dictaduras, de reivindicación de derechos políticos. Si bien la producción en esta línea tendió a decaer en los años siguientes, a finales de los '90 nuevamente volvieron a adquirir cierto protagonismo. En esta nueva oleada, sobre todo en los últimos años, ha existido un especial interés por estudiar la participación de mujeres en movimientos vinculados al medioambiente.

La evidencia muestra que principalmente durante los años ' 90 , la movilización de las mujeres "se produce a partir de situaciones ligadas a la vida cotidiana y a la experiencia y conocimientos medioambientales derivadas de la misma" (Sabaté, 2000: 


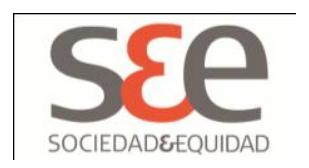

181). Ellas se hacen partícipes de innumerables acciones dispersas por todo el mundo, referidas a la defensa de los recursos naturales, la protección de la salud de sus hijos(as) y del entorno, la utilización de energías limpias y renovables, iniciativas de producción ecológica y sostenible. Contando solo a América Latina, son varias las experiencias de participación de mujeres ligadas a conflictos medioambientales. La resistencia a los monocultivos de pinos en las zonas de Pichincha y Esmeralda en Ecuador, contra los monocultivos de eucaliptos en el estado de Espíritu Santo en Brasil, contra los efectos de la cría de camarones en Ecuador, contra la instalación de represas en el sur de Chile, son solo algunos ejemplos de dicha realidad (Quesada, 2011).

La activa participación de las mujeres en este tipo de movimientos se debería a que ellas han sido uno de los colectivos más maltratados con los procesos de globalización económica y sus políticas de liberalización y flexibilización: “el sistema de liberalización de los mercados y servicios sigue apoyándose en el trabajo no retribuido realizado por las mujeres, tanto de reproducción en el ámbito familiar como comunitario, todo lo cual mantiene y refuerza las estructuras del patriarcado" (Sabaté, 2000: 323$)^{2}$.

Los cambios en la agricultura por las políticas de la Organización Mundial del Comercio $(\mathrm{OMC})$ han implicado la pérdida de control sobre la producción de ciertos recursos alimenticios, y dado que las mujeres son en general quienes se erigen como las responsables del cuidado y reproducción de la unidad doméstica, se ven mayormente afectadas. La privatización y regulación por medio de patentes les ha hecho perder control sobre recursos que utilizan no solo para alimentación, sino también para medicina natural, cuestión que sumada a la disminución de servicios sociales y a los mayores niveles de contaminación producto de la agroindustria, actividad minera, etc., ha implicado mayor sobrecarga de trabajo para ellas, pues se ha elevado la cantidad personas enfermas a las que deben cuidar (Quesada, 2011).

Otro de los ámbitos en que se han visto más afectadas las mujeres es respecto a la propiedad de la tierra. Específicamente para el caso de América Latina, un estudio realizado por Deere y León (2004) sobre las reformas agrarias realizadas desde la década de los '70 en adelante, demuestra que si bien con la llegada del neoliberalismo se generaron ciertos mecanismos que han facilitado el acceso a la tierra para las mujeres, los logros conseguidos se han debido más a la presión del movimiento de mujeres y feminista -que se consolidó en la región durante el mismo período- que a las bondades

${ }^{2}$ La cursiva es de la autora. 


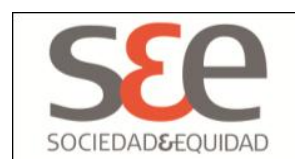

inherentes del sistema. Según FAO (2011), en las “regiones en desarrollo" las mujeres poseen en promedio menos explotaciones que los varones y tienen menos acceso a los distintos recursos e insumos agrícolas.

Debido a las cuestiones anteriormente señaladas es que se ha observado que en toda variedad de luchas vinculadas al medio ambiente en que participan las mujeres, ellas tienden a sumarse a aquellas experiencias en que se está apostando por un nuevo modelo de producción y desarrollo, y donde la temática de la alimentación y la agricultura son ejes claves de transformación. Justamente este es el caso del movimiento internacional La Vía Campesina (LVC), el cual fue fundado en 1993 por distintas organizaciones de campesinos, agricultores de pequeña y mediana escala, mujeres del campo, trabajadores agrícolas y comunidades agrícolas en América, África, Asia y Europa. Este movimiento nació para resistir y oponerse a las políticas neoliberales, en particular a las de la OMC.

Desde sus inicios la LVC ha tenido una especial preocupación por la mujeres y los temas de género (Velasco, 2011; Desmarais, 2007). Actualmente es el propio movimiento quien reafirma este interés: "Las mujeres juegan un papel fundamental en el trabajo de La Vía Campesina. Según la FAO, las mujeres producen el $70 \%$ de los alimentos mundiales pero están marginadas y oprimidas por el neoliberalismo y el patriarcado. El movimiento defiende los derechos de las mujeres y la igualdad de género a todos los niveles y lucha contra todas las formas de violencia hacia las mujeres" $<w w w$.viacampesina.org $>$. Este reconocimiento explícito por la temática de género es justamente lo que convierte a este movimiento en un objeto de estudio interesante.

Sin embargo, cabe señalar que a pesar de este estallido de influencia de la participación de las mujeres en movimientos medioambientales, y en general en todos los movimientos sociales, en especial en aquellos críticos contra el modelo social y económico, su activismo no ha sido una cuestión fácil de llevar. Tanto las experiencias pasadas como las más actuales evidencian que las dificultades y tensiones que se han dado al interior de los movimientos se incrementan a medida que las mujeres comienzan a demandar mayores cuotas de responsabilidad y poder (Dunezat, 2006).

De hecho, en general se ha observado que en los movimientos que enarbolan una ideología de "izquierda" ha primado la visión política de Lenin (1971), en que la "liberación de las mujeres" queda supeditada a la modificación de las bases materiales y a la transformación del modelo de producción, quedando subordinado de forma simplista el conflicto de género al de clase. Según Rauber (2005), esta perspectiva olvida las 


\section{SSe}

propias tesis planteadas por Marx y Engels sobre la relación entre el modo de producción capitalista y la subordinación de las mujeres. De hecho señala respecto a la interpretación que ha hecho "la izquierda":

Es curioso notar que tales planteamientos quedaron relegados o directamente desconocidos por las corrientes predominantes del marxismo dogmático bajo el prisma reduccionista y mecánico, hicieron de la explotación económica un problema exclusivo de la clase obrera industrial (...), y de la economía un ámbito separado de lo social y la cultural. La izquierda formada mayoritariamente en este pensamiento hizo de la problemática de la discriminación y explotación familiar y la explotación socioeconómica de las mujeres, una cuestión particular, una "contradicción secundaria" del capitalismo (Rauber, 2005: 14).

Este tipo de interpretación ha llevado al pensamiento tradicional de izquierda a separar la cotidianidad del quehacer político, y a supeditar los conflictos de género al económico, acusando a las causas feministas de burguesas o de falta de visión política al querer también dar un rol protagónico a una "contradicción" y lucha secundaria, la que sería resuelta luego de haber ganado la lucha contra el capital.

Esta perspectiva que ha generado variadas tensiones con las secciones femeninas dentro de los movimientos, también se observan en movimientos de carácter étnico. Según Arnold y Spedding (2006), la misma posición adoptada por las ideologías de izquierda que aseveran que una vez lograda la victoria del proletariado la desigualdad de género desaparecerá, ha sido heredada por algunos sectores de los movimientos campesinos e indígenas en América Latina (más marcadamente en estos últimos), "con la diferencia de que se arguye el colonialismo, y no la burguesía, como el enemigo principal, y no se deben desviar esfuerzos de combate hacia cuestiones de relaciones como las de género- que pueden fomentar desigualdades y exclusión dentro de los mismos grupos indígenas" (Arnold y Spedding, 2006: 23).

Es debido a estas cuestiones que esta investigación buscó analizar cómo se ha ido integrando la perspectiva de género en un movimiento social específico, teniendo en cuenta que durante los últimos años la participación de las mujeres se ha concentrado en movimientos relacionados al medio ambiente, la agricultura y diversas experiencias que apuestan por nuevos modelos de desarrollo. De ahí que se optara por hacer de la 


\section{SEe}

experiencia de La Vía Campesina el foco de estudio, sobre todo porque este es un movimiento que ha reconocido explícitamente la importancia del enfoque de género en su programa.

\section{ASPECTOS METODOLÓGICOS}

\section{Sobre el foco de estudio y las unidades de análisis}

Al ser un movimiento transnacional, la LVC tiene una estructura compleja, la cual se divide en 8 regiones ${ }^{3}$. En el caso de Latinoamérica las distintas organizaciones se han reunido en la Coordinadora Latinoamérica (CLOC), la que congrega organizaciones de América Latina y el Caribe. El foco de análisis de esta investigación recayó en la sección Latinoamérica (CLOC), debido a que ésta -considerando a Sudamérica, Centroamérica y el Caribe- representa el bloque más fuerte dentro del movimiento, con 66 organizaciones de un total de $148^{4}$.

Las máximas instancias en que se definen las direcciones, políticas y estrategias globales del movimiento son las Conferencias Internacionales de LVC, y en las Asambleas de Mujeres, en la que cada tres o cuatro años se reúnen delegados de todas las regiones. La CLOC, siguiendo el modelo de LVC global, realiza antes de las Conferencias Internacionales congresos y Asambleas de Mujeres a nivel regional.

La investigación desarrollada se basó en el análisis de textos (escritos y audiovisuales) producidos por el propio movimiento LVC, los cuales constituyeron las unidades de análisis del estudio. En específico se analizaron las declaraciones generales de las Conferencias y Asambleas de mujeres Internacionales de la LVC ${ }^{5}$, y las declaraciones de los Congresos de la Coordinadora Latinoamericana de Organizaciones Campesinas (CLOC), y dentro de éstas, se incluyeron declaraciones generales de las Asambleas de Mujeres y de las de Juventud. Además se analizaron documentos audiovisuales de LVC a nivel internacional y de la sección Latinoamérica (CLOC).

3 África, Europa, Centroamérica, El Caribe, Sudamérica, Norteamérica, sur de Asia y Este y Sudeste de Asia.

${ }^{4}$ Información obtenida de la página de LVC: http://viacampesina.org, y válida para Julio del 2011.

${ }^{5}$ Para que sirvieran de comparación con el fin de identificar las particularidades de América Latina. 


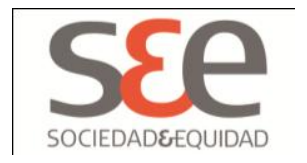

\section{Sobre el marco metodológico}

La metodología utilizada en esta investigación se basó en el análisis de marcos (frame analysis), ya que ella posibilitaba poner en evidencia tanto los elementos ideológicos como culturales que median en la construcción del movimiento (Zald, 1999). Hunt, Benford y Snow (2001), basándose en los análisis de marcos de referencia elaborados por Goffman (1974), adaptaron su enfoque a los movimientos sociales para indagar cómo ellos confieren sentido a sus mundos sociales y definen las metas y objetivos que los orientan.

Los "marcos de referencia" aluden a esquemas interpretativos sobre los cuales se codifican ciertos acontecimientos de la realidad. Dentro de los marcos de referencia se identifican dos tipos: unos llamados marcos maestros, que aluden a modelos de interpretación más universales y menos supeditados a un contexto específico, basados en una serie de valores compartidos; y otros llamados marcos de la acción colectiva en que se promueve directamente la movilización (Johnson, 2002).

Cuando se trata de movimientos sociales, los "marcos de acción colectiva" no solo destacan ciertos aspectos de la realidad, sino que también actúan como base para la atribución y articulación de significados. Según Hunt, Benford y Snow (2001), en el proceso de construcción de marcos necesarios para que un movimiento alcance el consenso que posibilite la movilización colectiva, se distinguen tres tareas fundamentales: la creación de marcos de diagnóstico, de pronóstico y de motivación. De forma correlativa señalan que a medida que se generan estos marcos, también se construyen una serie de diferentes posiciones, denominadas operacionalmente como campos de identidad, que corresponden a: antagonistas, audiencias y protagonistas.

En el "marco diagnóstico" se identifican tanto los acontecimientos y situaciones problemáticas y necesitadas de cambio como los agentes responsables de esta situación: los antagonistas. En el "marco pronóstico" se establece un plan para corregir la situación problemática, especificando los objetivos, tácticas, estrategias (repertorios de protesta) y actores susceptibles de ser alcanzados por los mensajes y acciones del movimiento: las audiencias. Pero aunque ambos marcos -diagnóstico y pronóstico- son necesarios para la generación del movimiento, por sí mismos no son suficientes para que se logre pasar del consenso de la situación de injusticia que se vivencia a la acción, pues para ello además se requiere de un conjunto de razones suficientes y apremiantes que justifiquen la acción a favor de la causas que se propugnan; dichas razones conforman el 


\section{SEe}

"marco de motivación" y ayudan a conformar el "nosotros" de la acción, es decir, el campo de los protagonistas.

Por lo tanto, marco motivacional/protagonistas, marco diagnóstico/antagonistas y marco pronóstico/audiencias, configuraron las tres categorías sobre las cuales se estructuró el análisis.

\section{EL ANÁLISIS DE CONSTRUCCIÓN DE MARCOS DE LVC}

\section{Marco motivacional: ¿Quiénes son La Vía Campesina?}

\section{Las y los protagonistas de la lucha}

La Vía Campesina se autodefine como un movimiento compuesto por diferentes personas "vinculadas a la tierra", de diversas culturas y países. Es posible identificar a través de los textos cómo el "nosotros" que define al movimiento ha ido cambiando a través de los años.

De ser un "nosotros" compuesto de campesinos y pequeños agricultores de todas las regiones del mundo, ha pasado a ser un "nosotr@s" mixto y diverso de "organizaciones de mujeres rurales, campesinos y campesinas, pequeños agricultores y agricultoras, trabajadores y trabajadoras del campo, pueblos indígenas, afrodescendientes y juventud rural, de Asia, Europa, América y África” (LVC, 2008: $\left.\mathrm{d} 8^{6}\right)$.

Quienes conforman este movimiento se erigen como los y las protectoras de la vida, y ese rol les llama a luchar y enfrentarse al sistema económico actual que pone la vida en peligro. Respecto de los principios motivacionales que van dotando de una identidad particular a sus miembros, en primer lugar se evidencia la existencia de un compromiso y responsabilidad tanto con el bienestar de la humanidad como del medio ambiente, proyectando una especie de ética del cuidado donde se busca el bienestar tanto de las personas como del medio ambiente.

Otro principio que se identifica es el resguardo y reconocimiento de la diversidad tanto genética como cultural. El respeto por los derechos e identidad de todos los

\footnotetext{
${ }^{6}$ Para no ensuciar el texto se indica con las siglas $\mathrm{dx}$ el documento específico al que se hace alusión. El detalle se encuentra en la bibliografía.
} 


\section{SSe}

pueblos y culturas, la igualdad de género y la autonomía tanto a nivel personal como comunitario se suman a esta lista. En casi todas las declaraciones, tanto de las conferencias internacionales como congresos a nivel latinoamericano, aparecen de forma reiterada los conceptos de_"igualdad", "diversidad” y "autonomía”, apuntando todos ellos como valor final a la justicia. En este sentido es posible observar que en el movimiento converge una ética del cuidado con una ética de la justicia entre todos sus participantes. La ética del cuidado asume un carácter transgenérico (Puleo, 2008), pues el cuidado del medio ambiente, el bienestar de las personas, la alimentación como acto de amor y la conexión con la Madre Tierra emergen como algo propio y distintivo de la identidad del movimiento en su conjunto y no solo de las mujeres.

En América Latina, más allá de estos principios motivadores que se comparten con el movimiento global, emerge también otro elemento esencial que también se constituye como un factor identitario clave, y que corresponde a la mística del movimiento. En la sección Latinoamérica existe una fuerte identificación con una mística propia, asociada a un imaginario que remite a esa "América Latina Rebelde" que resiste y lucha contra las injusticias, que está a favor de los más débiles y en contra de las fuerzas externas que han querido conquistarla. Esta mística se representa a través de íconos masculinos: Ché Guevara, Emiliano Zapata, Subcomandante Marcos, Simón Bolívar, José Martí, Augusto C. Sandino; todos reivindicados por distintos proyectos políticos de “izquierda".

Las mujeres no quedan exentas a esta mística y también se identifican con esos héroes masculinos que son referencia para su lucha. Probablemente esta identificación de las mujeres con los héroes masculinos responda a que el imaginario construido y socializado por la izquierda latinoamericana ha tendido -al igual que el resto de la sociedad- a invisibilizar la participación de las mujeres en los diversos procesos sociopolíticos, y en caso de destacarla, aparece siempre en su roles tradicionales clásicos. Por lo tanto, el "rostro de la revolución" ha sido preponderantemente masculino. Apelar a la rebeldía, al espíritu de lucha y de insubordinación implica reivindicar e identificarse con esos héroes.

Sin embargo, a pesar de esta preeminencia de referentes masculinos, también se observa que durante los últimos años se ha introducido en LVC un lenguaje inclusivo en que no solo se habla de los "héroes" de América Latina, sino también de las "heroínas"; y aunque el personalizar a los héroes y no a las heroínas sigue siendo la tendencia general, se van observando avances que van en sintonía con la propia mística con la que se pretende identificar la sección latinoamericana: “Trabajaremos por una mística 


\section{SEe}

latinoamericana que supere todo tipo de discriminación y violencia contra la mujer, facilitando y apoyando su participación activa y con iguales derechos que el hombre en las diferentes instancias de decisión” (CLOC, 1994: d11).

\section{Marco diagnóstico: La construcción “del problema” en La Vía Campesina}

\section{Los problemas a los que se enfrenta La Vía Campesina}

Los diversos problemas concretos a los que se aluden en los documentos revisados podrían agruparse en dos dimensiones:

1) Dimensión material: incluyen todos aquellos problemas que estarían afectando y condicionando directamente las condiciones de vida y supervivencia de las comunidades rurales y campesinas, y a trabajadores(as) ligados a la producción agrícola.

2) Dimensión cultural-simbólica: incluyen aquellos problemas referidos a la pérdida de los saberes propios de cada pueblo, como ciertas prácticas, cosmovisiones y otros elementos identitarios que los definen.

Sin embargo, más allá de esta distinción analítica en que podrían agruparse los problemas, se observa que en el contexto de este movimiento situaciones como la violencia de género, la migración (forzada), el deterioro del medio ambiente y la falta de autonomía, constituyen problemas que evidencian una estrecha relación entre lo material y lo cultural-simbólico. Por ejemplo, el deterioro del medio ambiente, producto del sistema de producción agroindustrial, no solo constituye un problema que les afecta de forma material directa por la contaminación del agua o la destrucción del suelo para siembras, puesto que para los y las campesinas -sobre todo de culturas indígenas- la naturaleza representa el medio que los provee de la vida y les posibilita su subsistencia. En este sentido, su expolio no solo constituye un problema material, que sino también atenta contra sus cosmovisiones, en las cuales se concibe una relación de armonía, complementariedad y respeto con -tal como la denominan ellas/os- La Madre Tierra. De este modo, estas concepciones estarían en la línea de una de las corrientes agrupadas bajo lo que se conoce como el "feminismo del Tercer Mundo", y dentro del cual el "ecofeminismo" de Vandana Shiva es el más destacado. Mies y Shiva (1997) señalan que se debe tener en consideración que para muchas mujeres del llamado Tercer Mundo, la división entre lo espiritual y material resulta incompresible, al igual que la división entre las llamadas “necesidades básicas” (alimento, cobijo, abrigo, etc.) 


\section{SEe}

y las llamadas "necesidades superiores" (como la libertad, el saber, etc.), pues para ellas la cultura formaría parte de la lucha por la subsistencia y la vida. En esta línea proponen un ecofeminismo que parta de las necesidades fundamentales de la vida, asumiendo lo que las autoras llaman una perspectiva de la supervivencia.

Ahora bien, más allá de que los problemas que se identifican en el movimiento, y que según sus cosmovisiones se ubican en una dimensión material-simbólica, cabe preguntarse si ellos tienen o no que ver con el género. Se observó que desde las primeras a las últimas Conferencias Internacionales y Congresos Latinoamericanos, cada vez más se fueron especificando los problemas concretos que vivencian las mujeres campesinas y rurales como colectivo. Si en las primeras declaraciones se hablaba generalmente de los problemas que padecían las familias campesinas, actualmente ya se explicitan ciertas problemáticas que afectan de forma directa a las mujeres. La violencia y el femicidio constituyen los principales problemas de género que se abordan en las declaraciones. Las limitaciones en la participación y una sub-representación en cargos de poder son otros de los problemas de género que han tenido cabida en las declaraciones. Además, durante los últimos años se ha explicitado cómo fenómenos como la pobreza y migración afectan de forma más perjudicial a las mujeres.

Por cierto, aunque se observa que se ha ido dando mayor visibilidad a los problemas de género en las "Declaraciones Finales", es necesario señalar, por un lado, que en dichos documentos el protagonismo sigue estando centrado en problemas estructurales derivados del modelo de agroindustria que afectan al campesinado en su conjunto, y por otro lado, que esto es mayor en los documentos que emergen de instancias propiamente de mujeres. En éstas instancias, tanto a nivel latinoamericano como internacional, se constata que no solo constituyen un problema para ellas las circunstancias que dificultan el cumplimiento de su rol como madres, en tanto responsables de asegurar la subsistencia de la unidad doméstica, sino también aquellas que las afectan de forma personal en su condición de mujeres, como por ejemplo lo referido a su participación socio-política. Son reiteradas las alusiones a la subrepresentación en los puestos de mayor peso político y liderazgo: “La reproducción del machismo y opresión dentro de las organizaciones de izquierda (partidos y movimientos, etc.), que hieren la dignidad de la mujer indígena, negra y campesina" (CLOCResolución, 1994: d12).

Otros problemas que denuncian las mujeres son la baja autoestima producto de la situación de subordinación en la que se encuentran, la falta de oportunidades para desarrollo personal y formación, y la violencia de género, entre los más mencionados. 


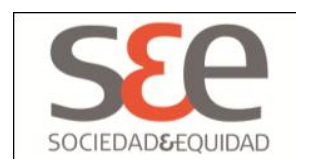

Además, señalan otras cuestiones que les afectan en tanto trabajadoras/productoras, como la discriminación salarial, dificultades para disponer de créditos o para acceder a la titularidad de tierras. En suma, cabe decir que sus problemas, más que problemas de mujeres, son problemas derivados, en su gran mayoría, de la estructura de género hegemónica, ante la cual ellas no son ciegas. De todos modos, es necesario destacar que las mujeres no solo denuncian problemas referidos al género, sino que también son parte de su diagnóstico aquellas problemáticas de tipo más estructural, económicas y culturales que afectan al campesinado y sectores rurales en su totalidad.

\section{Los ejes de conflicto: las causas de los problemas}

Se identificaron tres ejes de conflictos que emergen como las principales causas de los diversos problemas concretos que denuncia La Vía Campesina, aunque ellos no tienen el mismo peso según la sección y grupo al que se haga alusión.

El eje de conflicto dominante que se identifica tanto en los documentos de las Conferencias Internacionales como de los Congresos CLOC y las diversas Asambleas de Mujeres, es el capitalismo neoliberal; éste se constituye como la principal causa de los problemas que enfrenta el campesinado y los sectores rurales en general. Es dicho sistema el que ha condicionado la conversión a un sistema agroindustrial, y que a su vez ha generado hambre, pobreza, deterioro ambiental y otros problemas sociales que afectan a miles de personas. El capitalismo neoliberal es el principal eje de conflicto sobre el cual el movimiento erige su lucha.

Otro eje de conflicto que se identifica en el movimiento -aunque con mayor preponderancia en la sección latinoamericana que en la internacional- es el colonialismo, el cual junto con el capitalismo es considerando como el principal causante de la destrucción de los saberes locales, cosmovisiones y elementos propios de cada cultura. La colonización no solo se habría dado en término físicos, sometiendo y esclavizando a miles de indígenas y nativos de América Latina en la época de la conquista europea, sino también se habría extendido a través de los años, trasponiendo cosmovisiones, imponiendo una mentalidad menos sostenible y desvalorizando los conocimientos locales.

Un tercer eje de conflicto que se identifica en los textos es el patriarcado. Este eje se destaca principalmente en los documentos que emergen específicamente de las Asambleas de Mujeres (tanto internacionales como latinoamericanas), aunque ya en las 


\section{SEe}

Declaraciones Finales de las Conferencias Internacionales y Congresos CLOC no tienen el mismo peso.

Estos tres ejes de conflicto (capitalismo, colonialismo y patriarcado) se identifican particularmente en los planteamientos que emergen de las Asambleas de Mujeres de la sección Latinoamérica. Esto es de suma importancia, puesto que concentrarse no solo en la crítica anticapitalista y eurocéntrica, sino también patriarcal, les permite identificar tanto causas externas como internas a las que se deben enfrentar para modificar su condición de subordinación, teniendo en cuenta que además estas últimas no solo se reproducen en el sistema en general sino también dentro de sus propias culturas y comunidades. A pesar de ello, aún se observa cierta tendencia a poner mayor acento en los problemas derivados de causas que se consideran externas, como el capitalismo y colonialismo. De hecho más allá de los temas de representación política y violencia de género, se observa una menor problematización acerca de cómo las mujeres, sus compañeros y sus prácticas diarias reproducen el sistema patriarcal. Sin embargo tampoco se observa que hayan caído en una completa idealización de sus culturas donde se pasen por alto los problemas de género internos. En este sentido, aunque quizás aun de forma muy incipiente, se observa que planteamientos como los de Puleo (2008), referidos a la necesidad de no solo erigir la crítica sobre la racionalidad occidental europea como única causante de los problemas que enfrentan las mujeres, la naturaleza y el medio ambiente, sino también la necesaria revisión de los prejuicios patriarcales presentes en culturas que pueden ser más ecológicamente sostenibles, tienen cabida en este movimiento, en particular entre las mujeres, pues aunque se apela al reconocimiento y diferencia cultural, no caen en un relativismo acrítico de las desigualdades de género.

Volviendo a las relaciones entre los distintos ejes de conflicto, la relación entre capitalismo y patriarcado es más evidente para las mujeres, ya que señalan que dada sus propias condiciones históricas, los efectos del sistema son más perjudiciales para ellas. En el sistema económico actual, que tiene como meta aumentar la producción y valora más los productos que pueden ser comercializados, desvaloriza los trabajos reproductivos y regenerativos que históricamente han estado mayormente en manos de las mujeres.

En suma, se observó que existe cierta diferencia entre el movimiento en general y las secciones de mujeres, pues en el primero aun el patriarcado aparece como un eje menos determinante que el sistema económico. Sin embargo, el definir el capitalismo como el principal eje de conflicto, no ha impedido que emerjan otros ejes de lucha 


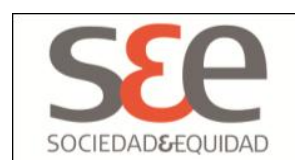

distintos, los cuales aunque aun no han logrado permear a todos los miembros, en especial a los varones, tampoco han quedado totalmente invisibilizados. Más bien se observa cómo con el pasar de los años, a medida que se trabajan más los problemas de género y la definición del patriarcado como eje de conflicto en las Asambleas de mujeres, se ha ido logrando permear gradualmente al resto del movimiento.

\section{Antagonistas: los responsables de los problemas}

Los principales responsables que aparecen de forma más clara e identificable, en LVC global y a nivel de CLOC, son tanto los organismos internacionales como las empresas transnacionales. Los primeros, entre los cuales caben FMI, Banco Mundial, OMC, organismos de Naciones Unidas e incluso FAO, han promovido políticas y medidas que han favorecido el avance del capital financiero, es decir, a las empresas transnacionales, las cuales a su vez solo tienen como objetivos maximizar sus beneficios, sin importarles el bienestar de las personas, pueblos y países.

Respecto a los problemas de género que se denuncian, los responsables emergen de forma mucho más soterrada. Ya se ha mencionado anteriormente que existe cierta tendencia a buscar responsables en el ámbito externo más que interno. Sin embargo, aunque se ha identificado como responsables a los Estados o al propio sistema económico, también se considera la responsabilidad de las propias comunidades, organizaciones y compañeros en la reproducción de ciertos problemas relacionados con el género.

\section{Marco pronóstico: “la solución” al problema}

\section{Objetivo y soluciones}

En total sintonía con el diagnóstico realizado por el movimiento, en que el sistema capitalista neoliberal se erige como una de las principales causas de los diferentes problemas que los aquejan, se presenta su desarticulación como uno de sus objetivos más importantes. Tanto a nivel internacional y latinoamericano, como en las instancias específicamente de mujeres, se comparte el objetivo de "confrontar y vencer a la agenda global del neoliberalismo” (Vía Campesina, 2000: d2).

Es pertinente preguntarse si los objetivos que se propone el movimiento abordan los problemas de género planteados y si interpelan al sistema patriarcal que también se había diagnóstico como una causa del problema. Al igual como sucedía en el proceso de 


\section{SEe}

construcción del marco diagnóstico, es en los documentos que emergen de las Asambleas de Mujeres donde se hace explícito el objetivo orientado a modificar el sistema que reproduce las desigualdades de género. En este sentido, en dichos documentos no solo se llama a desarticular el sistema económico, sino también el patriarcal, restableciendo relaciones más simétricas entre los sexos. Particularmente en los Congresos de la sección Latinoamérica hay constantes alusiones al compromiso de contemplar la igualdad de género en todas las reformas integrales que se lleven a cabo, y a propiciar la participación de todos los actores en igualdad de condiciones: "Nuestro proyecto busca construir y consolidar un poder popular incluyente y democrático en el que participen todos los actores sociales involucrados, mujeres, jóvenes, niños, pueblos indios, a partir de sus propias reivindicaciones, reforzando desde sus bases cambios profundos en los aspectos económicos, sociales, políticos y culturales" (CLOC, 2001:d15)

Por su parte, en las asambleas de mujeres sí se determina como objetivo concreto la lucha contra el patriarcado, además de proponer como objetivo no solo su desarticulación, sino también la construcción de una nueva sociedad erigida sobre nuevas relaciones de género, donde esto no solo implique un cambio en la mentalidad de los varones sino también en la de las propias mujeres: "Debemos luchar por la nueva mujer, el nuevo hombre, las nuevas relaciones en una nueva sociedad" (CLOC, 1994: d2).

Enmarcado dentro de los preceptos de su propia cosmovisión del "Buen Vivir", en que priman los conceptos de complementariedad, consenso, reciprocidad y armonía, definen como su principal objetivo el generar una sociedad basada en esos principios: "La América Latina que queremos es una que se construya de relaciones armoniosas y de interdependencia entre seres humanos, constituidos como iguales, que encaminen su accionar a la luz de la sostenibilidad de la vida" (Asamblea de Mujeres-CLOC, 2010:d21). Sin embargo, esto en ningún caso implica que se pasen por alto los conflictos de género existentes.

Ahora bien, respecto de las soluciones para hacer frente a los problemas y sus causas diagnósticas, el movimiento propone una solución integral: la soberanía alimentaria, la cual daría una solución global a las crisis múltiples que se enfrentan actualmente. Para que sea posible el desarrollo de la soberanía alimentaria, se requeriría del cumplimiento de los derechos de los pueblos indígenas y campesinos, del reconocimiento de sus saberes y conocimientos, de sus derechos de participación en igualdad de condiciones y de la prevalencia como ejes claves de principios como la sostenibilidad, el respeto, la autonomía y la igualdad. 


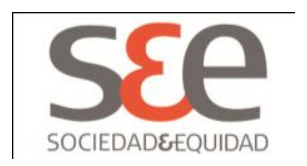

Las mujeres del movimiento, justamente apelando a estos principios, han mostrado que la soberanía alimentaria tampoco será posible sin el reconocimiento del rol clave que ellas juegan en este proceso. Tampoco sería posible si no se contempla la igualdad de género tanto en la construcción del nuevo modelo como dentro de las propias organizaciones y el movimiento en general. Ellas han demandado que se contemple la igualdad de género en todas las reformas, actividades, estrategias y decisiones que se requieran para lograr la soberanía alimentaria. El movimiento, tanto en su sección Latinoamérica como internacional, ha hecho eco de estas demandas.

En América Latina, la solución de la soberanía alimentaria se ha enmarcado en la construcción de un modelo socialista. Sin embargo, desde las Asambleas de Mujeres regionales también se reivindica la necesidad de avanzar hacia la construcción de un modelo socialista pero de la mano de un proyecto feminista, bajo el lema de "Sin feminismo no hay socialismo". Así, se progresa cada vez más tanto en la visibilización como integración de la perspectiva de género en el movimiento. Esta cuestión marca un punto de inflexión debido a que el reconocimiento, e incluso la utilización de la palabra "feminismo", marca una diferencia entre las últimas asambleas y las realizadas anteriormente, donde se hablaba de la lucha contra el patriarcado, pero no se reconocía explícitamente como una lucha feminista. Sin embargo, no se prioriza ni el proyecto feminista ni el socialista, puesto que se entiende que ambos están relacionados y que se necesitan mutuamente para conseguir los objetivos propuestos.

Es interesante resaltar cómo desde el primer Congreso y Asamblea de Mujeres a nivel latinoamericano se han ido introduciendo diversos conceptos, lenguajes y problemas que evidencian un mayor grado de concientización respecto al género. Llegar a reconocer su lucha como una lucha feminista es un ejemplo de ello, puesto que más allá de las particularidades que asuma su proyecto feminista, el mero hecho de aludir a ese concepto remite a un imaginario determinando, el cual por sí mismo ha generado variadas resistencias, sobre todo en sectores indígenas y rurales latinoamericanos.

\section{Roles en la solución del problema}

Respecto a los roles que asumen los distintos actores en la solución de los problemas diagnosticados, se evidencia que en particular las mujeres vivencian procesos paralelos en los cuales, por un lado, cuestionan los roles y posiciones de género asignados patriarcalmente, intentando modificarlos, y por otro, se reproducen ciertas 


\section{Sce}

concepciones que -leídas desde el "feminismo occidental"- promueven las persistencia de ciertos prejuicios patriarcales.

Se evidencia entonces un proceso complejo en que, por un lado, se reivindican y perpetúan roles tradicionales, pero ellos mismos se utilizan como fundamento para introducir cambios y transformaciones que las favorezcan. El tener el rol histórico de proveedoras, cuidadoras, protectoras del medio ambiente y de su biodiversidad es justamente aquello que a su vez les permite reclamar nuevas posiciones de poder respecto de los varones. Esto sería posible debido principalmente al hecho de que justamente en la solución que propone La Vía Campesina se reivindica un modelo de desarrollo y producción en el cual tanto el cuidado de la Naturaleza como de las personas, la preservación de la diversidad, el bienestar de las pueblos y la provisión de necesidades básicas -donde la más importante es la alimentación- constituyen ejes claves de su propuesta. Las mujeres exaltan ciertos roles, características y saberes, justamente porque son valorados dentro de la cosmovisión de la cual emerge su propuesta política.

Las mujeres de la Vía Campesina buscan asumir un rol protagónico en la lucha por conseguir la soberanía alimentaria. Abogan por el reconocimiento de sus conocimientos, saberes y prácticas como algo esencial para la consecución del fin que se han propuesto. Marcan las diferencias respecto de lo que ellas aportan en comparación con los varones, para desde ahí hacer escuchar y valer su voz.

\section{Estrategias y acciones}

- Participación de los actores involucrados: la apuesta por construir desde abajo. Se promueve la participación de los propios involucrados y afectados en la solución de los problemas. Si son las mujeres quienes además de sufrir de forma más perjudicial las consecuencias del modelo económico y del deterioro ambiental, es evidente que ellas también deben participar en igualdad de condiciones en la búsqueda de la solución. Es la misma estrategia que adopta el movimiento la que abre también las puertas a la participación de las mujeres, y genera que ellas reivindiquen esas instancias. Además, al apostar por una transformación del modelo de "abajo hacia arriba", donde el cambio es construido desde los actores mismos y desde su práctica concreta, les permite interpelar a una transformación que parte de sus propias relaciones personales.

- Alianzas, redes, articulación. Esta emerge como la principal estrategia del movimiento. Se reconoce la importancia de lograr sumar fuerzas para alcanzar los 


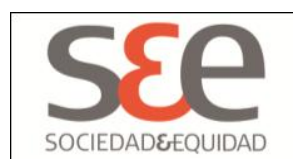

objetivos propuestos, y por eso se propicia la articulación con todos aquellos actores y movimientos que compartan su lucha. Esta estrategia posibilita tanto que las mujeres se comprometan en la lucha general del movimiento como en la lucha contra el patriarcado. Las mujeres han sabido utilizar esta estrategia para generar puentes con los varones y hacer que sus planteamientos, poco a poco, se vayan incorporando como un problema no solo de ellas sino del movimiento en su conjunto; y aunque aun no se haya logrado una completa integración, se van identificando avances importantes.

- Educación y formación. Las mujeres y jóvenes son quienes más apelan a esta cuestión. Existe un reconocimiento general a nivel del movimiento de que ambos grupos han sido los más marginados dentro de los sectores rurales y campesinos. Y como se les asigna un rol clave en el proceso de transformación, su formación política y educativa se considera una estrategia clave.

- Transversalización del enfoque de género. Esta estrategia es promovida específicamente por las asambleas de mujeres, y busca que "Todos los miembros en todas las organizaciones participantes de La Vía Campesina tienen que aceptar la importancia de desarrollar una perspectiva de género, de clase y de etnia e integrarlo en sus cuadros de trabajo" (Vía Campesina-documento, 2000: d3).

Respecto de las acciones concretas que se han tomado en el movimiento bajo una perspectiva de género, se destacan las siguientes:

- Organización específica de mujeres. Aunque se apela a una lucha conjunta entre hombres y mujeres, se estipula la necesidad de trabajar en instancias específicas propias de mujeres, rechazando todos los supuestos que apuntan a ver en estas acciones el objetivo de dividir a las organizaciones mixtas.

- Paridad de género. Se ha establecido tanto a nivel nacional como internacional la participación de las mujeres en un $50 \%$ en todas las esferas e instancias del movimiento (conferencias, comisiones, eventos), y en particular en los espacios de decisión y dirección.

- Declaración de movilización permanente para combatir la violencia contra las mujeres.

- Debate sobre derechos sexuales y reproductivos. 


\section{SEe}

\section{Audiencia: a quién busca llegar el movimiento}

El movimiento La Vía Campesina busca alcanzar a una audiencia externa compuesta por aquellas personas, movimientos, organizaciones y sectores que se puedan sentir identificados con su lucha. Hay una intención de llegar a la mayor cantidad de audiencia $y$, en lo posible, lograr que de alguna manera se vinculen a su lucha.

Sin embargo, el movimiento no solo pretende impactar a una audiencia externa, sino también a una audiencia interna. Esto es de especial importancia para la lucha por la igualdad de género. Las mujeres buscan llegar tanto a sus compañeros varones como a las distintas organizaciones que componen el movimiento, y a la facción juvenil del mismo. Conseguir tener entrada, sobre todo en este último grupo, reviste especial interés para ellas, pues de allí saldrán los y las futuras líderes.

Por lo tanto, llegar a instalar entre ellos y ellas la perspectiva de género constituye una cuestión de interés estratégico para la lucha de las mujeres. También dentro de esa audiencia interna se busca llegar a todas aquellas mujeres que son parte del movimiento pero que aun no logran empatizar y concientizarse en las temáticas de género.

\section{CONCLUSIONES}

Es posible concluir que LVC ha ido integrando de forma gradual una perspectiva de género en la construcción de su marco de referencia, de su marco motivacional y en la construcción del sujeto protagonista. Precisamente este último estaría definido en función del reconocimiento de la diversidad. Si bien la identidad campesina es lo que dota del principal elemento aglutinador sobre el que se erige el "nosotros" del movimiento, ésta identidad actuaría más a modo de esencialismo estratégico que en términos reales. El reconocimiento de la diferencia es lo que justamente posibilita que las mujeres tengan visibilidad como sujetos específicos en este movimiento, y no queden subsumidas ni invisibilizadas bajo un sujeto protagónico monolítico.

En torno al marco diagnóstico y la construcción del problema, se observó que a diferencia de las interpretaciones más ortodoxas asumidas tradicionalmente por los movimientos de izquierda, que tienden a infravalorar otros ejes de conflicto distintos al económico, en este caso se apreció mayor dinamismo para incorporar nuevas problemáticas. Aunque el eje de conflicto predominante sigue siendo el sistema 


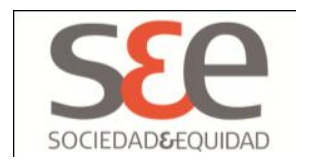

económico, se evidencia mayor apertura para ir incorporando otros nuevos, lo que erige a LVC en un movimiento bastante dinámico en lo que respecta a la construcción del marco diagnóstico. Asimismo, respecto a este marco diagnóstico cabe señalar como una potencial tensión el hecho de que, si bien hasta este momento se considera una fortaleza la permeabilidad del movimiento a incorporar los distintos problemas de cada grupo a su marco de referencia, podría llegar a constituirse en una posible amenaza para la causa de las mujeres, ya que se puede correr el riesgo de ir añadiendo las distintas reivindicaciones particulares de cada grupo bajo una lógica de mera agregación. Así, las problemáticas de género pasarían a ser una más de entre otras tantas, y se perdería el potencial actual que se observa de ir realmente integrando -aunque sea de forma gradual- los conflictos de género como un eje central, y no como un problema específico que se sumaría a una larga lista.

Por otro lado, respecto al marco pronóstico y la construcción de "la solución", se observó que ésta se encuentra construida en función de una propuesta -la soberanía alimentaria- que apuesta por un nuevo modelo de desarrollo basado en principios de igualdad, justicia, dignidad y autonomía, en el cual se contemplen todos los actores involucrados, en especial aquellos que han ido quedando marginados en el sistema actual. Asimismo, esta propuesta reivindica la imperiosa necesidad de poner en el centro aquellas cuestiones que son básicas para el mantenimiento de la vida. En este sentido, es una propuesta que parte de las experiencias concretas, apostando por una transformación global e integral, pero que comienza en lo cotidiano: cómo producir, qué producir, de qué alimentarse. Esta lógica posibilita que las mujeres integren la perspectiva de género, apelando al hecho de que para generar ese cambio y conseguir una transformación del sistema en su conjunto, es necesario en primer lugar cambiar lo cotidiano. La construcción de una sociedad más justa e igualitaria, solo sería posible si primero los principios de justicia e igualdad se concretizan a nivel interpersonal, tanto dentro de las propias familias como de las organizaciones y comunidades.

Se apela tanto a la autonomía de los pueblos como de las mujeres, y en ese sentido se busca recuperar aquello que se considera exterminado por causa de las fuerzas combinadas del capitalismo y colonialismo, pero mutatis mutandis respecto de la dominación patriarcal. La principal tensión respecto del marco diagnóstico -que se presenta más bien como pregunta- es si realmente la solución de la soberanía alimentaria, que busca restablecer una economía campesina, constituirá efectivamente una avance hacia un mayor empoderamiento de las mujeres. En este sentido, las tesis de Lenin (1971) referidas a la vinculación directa entre la economía doméstica campesina y la subordinación de las mujeres son importantes de considerar en el momento de 


\section{S\&e}

reivindicar la economía campesina. La mera restitución de una economía campesina, sin considerar la integración de una perspectiva de género, corre el riesgo de perpetuar ciertas desigualdades. Si no se logra avanzar en temas claves como la co-responsabilidad del trabajo doméstico y de cuidados de los miembros de la familia, por más que se gane con la economía campesina en autonomía e independencia del capital, las mujeres seguirán sobrecargadas de trabajo, al descansar en ellas tanto parte del trabajo productivo como doméstico. Lo mismo sucederá si no se logran fomentar las experiencias cooperativas entre mujeres y solo se priorizan unidades campesinas familiares, donde ellas sigan a cargo de la producción de subsistencia y los varones de lo productivo para el intercambio comercial. Tal como señalaba Lenin, tanto el intercambio de experiencias, la colectivización de sus problemas como la independencia económica constituyen factores claves de su emancipación.

Finalmente, solo cabe hacer referencia a la identificación de la gestación de un proyecto feminista dentro del movimiento La Vía Campesina. Este proyecto es bastante cercano a lo que Shiva y Mies (1997) llaman un "feminismo de la subsistencia", pues es un feminismo en que converge una lucha tanto por lo material como por lo simbólico, superando la división entre necesidades básicas y superiores. Así, se critica la hegemonía del pensamiento eurocéntrico que no reconoce los saberes y conocimientos de otras culturas, y que identifica todo "lo otro" como subdesarrollado. Además, prevalece la insistencia en una relación de cierta complementariedad y armonía entre los sexos, al igual que con la Naturaleza y el Medio Ambiente.

Sin duda una de las cuestiones más interesantes que se identifican en este movimiento es que actualmente existen ciertas condiciones que están posibilitando que no solo se desarrolle un proyecto feminista al interior de él, sino que también el propio movimiento asuma una identidad feminista. Solo resta esperar la próxima cosecha para ver si la semilla feminista dio frutos para todo el movimiento o solo alcanzó para las mujeres.

\section{Referencias Bibliográficas}

Arnold, Denise y Alison Spedding (2006), Género, etnicidad y clases sociales: la mujer en los movimientos sociales y movimientos de mujeres. En J. Espandín y P. Iglesias (Eds.), Bolivia en movimiento: Acción colectiva y poder. Barcelona: El Viejo Topo. [Documento en línea]. Disponible desde internet en: 


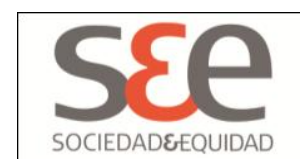

<http:www.redcimas.org/archivos/biblioteca/metodologias/DArnoldYASpedding_GENER O.pdf>

Deere, Diana y M. León (2004), Revertir la reforma agraria con exclusión de género: lecciones a partir de América latina. El Otro Derecho, 31-32 2004: 181-219.

Desmarais, A. (2007) La vía campesina: La globalización y el poder del campesinado. Madrid: Editorial Popular.

Dunezat, X. (2006), Luchas dentro de la lucha: Acción colectiva y relaciones sociales de sexo. Revista Política, $N^{\circ}$ 46, 2006: 227-249. [Documento en línea]. Disponible desde internet en:

<http: / / redalyc. uaemex.mx/redalyc/src/inicio/ArtPdfRed.jsp?iCve=64504609>

FAO (2010) El estado de la inseguridad alimentaria en el mundo. La inseguridad alimentaria en crisis prolongadas. Roma: Naciones Unidas.

Gamba, S. (2008) ¿Qué es la perspectiva de género y los estudios de género?. [Documento en línea]. Disponible desde internet en:

<http://www.nodo50.org/mujeresred/spip.php?article1395>.

Hunt, S., R. Benford \& D. Snow (2001), Marcos de acción colectiva y campos de identidad en la construcción social de los movimientos. En J. R. Gusfield \& E. Laraña (Eds.), Los nuevos movimientos sociales: De la ideología a la identidad (pp. 221-249). Madrid: Centro de Investigaciones Sociológicas.

Johnson, N. (2002), Uruguay. Agenda de género y articulación movimientos sociales. En Mundo paralelos. Agenda de género y movimientos sociales en Argentina, Chile y Uruguay (pp. 91-127). Programa Mujer y Democracia en el MERCOSUR. 


\section{SEe}

Lenin, V. (1971) La emancipación de la mujer: Recopilación de artículos. Moscú: Progreso.

Mies, M. \& V. Shiva (1997) Ecofeminismo: Teoría, crítica y perspectivas. Barcelona: Icaria.

Puleo, A. (2008), Libertad, igualdad, sostenibilidad. Por un ecofeminismo ilustrado. Isegoría. Revista De Filosofía Moral y Política. Feminismo, Nuevas Tendencias, $\mathrm{N}^{\circ} 38$, 1997: 39-59.

Quesada Guerrero, R. (2010), Empoderamiento de mujeres latinoamericanas a través de prácticas ecofeministas. Investigaciones Feministas, N¹, 2010: 97-109.

Rauber, I. (2005), Movimientos sociales, género y alternativas populares en Latinoamérica y el caribe. Itinéraires. Notes et travaux, $\mathrm{N}^{\circ} 77,2005$. [Documento en línea]. Disponible desde internet en:

<http://graduateinstitute.ch/webdav/site/developpement/shared/developpement/362 /itineraires\%20IUED/IUED_INT77_Rauber.pdf>

Sabaté, A. (2000), Género, medio ambiente y acción política: Un debate pendiente en la geografía actual. Anales de Geografía de La Universidad Complutense de Madrid, 20, 2000: 177-191.

Velasco Sesma, A. (2010), Justicia social y ambiental: Mujeres por la soberanía alimentaria. Investigaciones Feministas, 1, 2010: 161-176.

Zald, M. (1999), Cultura, ideología y creación de marcos estratégicos. En D. McAdam, J. McCarthy \& M. Zald (Eds.), Movimientos sociales: Perspectivas comparadas: 


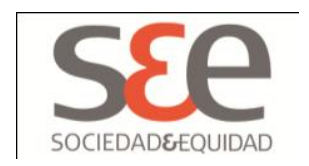

Oportunidades políticas, estructuras de movilización y marcos interpretativos culturales (pp. 369-388). Madrid: ISTMO.

\section{Páginas web}

www.viacampesina.org/sp/index.php?option=com_content\&view=category\&layout=blog \&id=27\&Itemid=44. Revisado 9 de julio de 2011.

\section{Textos internet de La Vía Campesina y CLOC}

\section{Documentos de las Conferencias Internacionales de La Vía Campesina.} La Vía Campesina (1996), Declaración de Tlaxcala de La Vía Campesina. México [documento en línea]. Disponible desde Internet en HTML en:

http://viacampesina.org/sp/index.php?option=com_content\&view=article\&id=404:iiconferencia-internacional-de-la-via-campesina-tlaxcala-mexique-18-al-21-abril1996\&catid=32:2-tlaxcala\&ttemid=48 [d1] La Vía Campesina (2000), Declaración IV Conferencia Vía Campesina en Bangalore. India. [documento en línea]. Disponible desde Internet en HTML en: http://viacampesina.org/sp/index.php?option=com_content\&view=article\&tid=326:decla racion-iv-conferencia-via-campesina-en-bangaloreftcatid=33:3-bangalore2000\&ltemid=55 [d2] La Vía Campesina (2000), Género. (Documento de la III Conferencia Internacional de Vía Campesina). Bangalore, India. [documento en línea]. Disponible desde Internet en HTML en:

http://viacampesina.org/sp/index.php?option=com_content\&view=article\&tid=325:gener oftcatid=33:3-bangalore-2000\&ltemid=55 [d3] La Vía campesina (2004), Declaración de IV Conferencia de La Vía Campesina. Itaici, Sao Paulo. [documento en línea]. Disponible desde Internet en HTML en:

http://viacampesina.org/sp/index.php?option=com_content\&view=article\&tid=52:declar acie-la-iv-conferencia-de-la-via-campesina\&catid=16:4-sao-paolo-2004\&ltemid=43 [d4] La Vía Campesina (2004), Declaración de la II Asamblea internacional de mujeres rurales. Itaici, Sao Paulo. [documento en línea]. Disponible desde Internet en HTML en: http://viacampesina.org/sp/index.php?option=com_content\&view=article\&tid=53:declar 


\section{SSe}

acie-la-ii-asamblea-internacional-de-mujeres-rurales\&catid=16:4-sao-paolo-

2004\&Itemid=43 [d5]

La Vía Campesina (2008), Carta de Maputo: V Conferencia Internacional de la Vía Campesina. Agricultura Campesina y Soberanía Alimentaria Frente a la Crisis Global. Maputo, Mozambique. [documento en línea]. Disponible desde Internet en HTML en: http: / / viacampesina.org/sp/index.php?option=com_content\&view=article\&id=620:cartade-maputo--v-conferencia-internacional-de-lavcampesina\&catid=45:declaraciones\&ltemid=70 [d6] La Vía Campesina (2008), Declaración III Asamblea de Mujeres en V Conferencia Internacional. Maputo, Mozambique. [documento en línea]. Disponible desde Internet en HTML en:

http: / /viacampesina.org/sp/index.php?option=com_content\&view=article\&id=608:decla racie-la-iii-asamblea-de-las-mujeres-lvc\&catid=45:declaraciones\&ltemid=70 [d7] La Vía Campesina (2008), Declaración de Maputo: V Conferencia Internacional de la Vía Campesina. Maputo, Mozambique, Octubre 19-22, 2008. Soberanía alimentaria YA! Con la lucha y la unidad de los pueblos!. Maputo, Mozambique. [documento en línea]. Disponible desde Internet en HTML en: http: / / viacampesina.org/sp/index.php?option=com_content\&view=article\&id=617:decla racie-maputo-v-conferencia-internacional-de-lavcampesina\&catid=45: declaraciones\&ltemid=70 [d8]

Documentos de los Congresos de la Coordinadora Latinoamericana de Organizaciones Campesinas (CLOC)

CLOC (1994), Declaración Final I Congreso, Perú. [documento en línea]. Disponible desde Internet en HTML en: http://www.cloc-viacampesina.net/es/congresos/i-congreso/218declaracion-final [d11] CLOC (1994), Resolución sobre el tema mujer, juventud y niñez, I Congreso. Perú. [Documento en línea]. Disponible desde Internet en HTML en: http: / /www.clocviacampesina.net/es/congresos/i-congreso/209-mujer-juventud-y-ninez [d12] CLOC (1997), Declaración de Brasilia, Il Congreso, Brasil. [Documento en línea]. Disponible desde Internet en HTML en: http://www.clocviacampesina.net/es/congresos/ii-congreso/245-declaracion-de-brasilia [d13] CLOC (2001), Declaración Final del III Congreso. México. [Documento en línea]. Disponible desde Internet en HTML en: http: / /www.clocviacampesina.net/es/congresos/iii-congreso/247-declaracion-final-del-iii-congreso[d14] 


\section{SEe}

CLOC (2001), Declaración II Asamblea Latinoamericana de Mujeres del Campo. México. [Documento en línea]. Disponible desde Internet en HTML en: http://www.clocviacampesina.net/es/congresos/iii-congreso/250-declaracion-de-la-ii-asamblealatinoamericana-de-mujeres-del-campo- [d15]

CLOC (2001), Declaración de la Juventud Rural Latinoamericana de la CLOC. I Asamblea Latinoamericana de Organizaciones del Campo. México. [Documento en línea]. Disponible desde Internet en HTML en: http://movimientos.org/cloc/show_text.php3?key=865 [ d16]

CLOC (2005), Declaración del IV Congreso de la Coordinadora Latinoamericana de Organizaciones del Campo. Guatemala. [Documento en línea]. Disponible desde Internet en HTML en: http://www.cloc-viacampesina.net/es/congresos/iv-congreso/251declaracion-final-del-iv-congreso- [d17] CLOC (2005), Declaración III Asamblea Continental de Mujeres. Guatemala. [Documento en línea]. Disponible desde Internet en HTML en: http: / /www.clocviacampesina.net/es/congresos/iv-congreso/253-declaracion-de-la-iii-asambleacontinental-de-mujeres- [d18]

CLOC (2005), Declaración II Asamblea de la Juventud Campesina. Guatemala. [Documento en línea]. Disponible desde Internet en HTML en: http: / /www.clocviacampesina.net/es/congresos/iv-congreso/252-declaracion-ii-asamblea-de-lajuventud-campesina [d19]

CLOC (2010), V CONGRESO DE LA CLOC: Declaración de Quito. Ecuador. [Documento en línea]. Disponible desde Internet en HTML en: http: / /www.clocviacampesina.net/es/congresos/v-congreso/noticias/402-clocomunicacoin [d20] CLOC (2010), IV Asamblea de la Articulación de Mujeres del Campo, CLOC - Vía Campesina, Declaración de Quito. Ecuador. [Documento en línea]. Disponible desde Internet en HTML en: http://www.cloc-viacampesina.net/es/congresos/vcongreso/noticias/389-iv-asamblea-de-la-articulacion-de-mujeres-del-campo-cloc-viacampesina-declaracion-de-quito [d21]

\section{Documentos audiovisuales}

Documental: La Vía Campesina en Movimiento... ¡Por la Soberanía Alimentaria!. [Documento en línea]. Disponible en: http://www.vimeo.com/27474387. [d9] Documental: V Conferencia Internacional Vía Campesina. Maputo, Mozambique. Vive TV. [documento en línea]. Disponible en: http://www.rebelion.org/noticia. php?id=92674. [d10] Documental: América Lucha. [Documento en línea]. Disponible para descarga en: http://www.4shared.com/account/dir/hKF1lVXY/_online.html?rnd=79 [d22] 


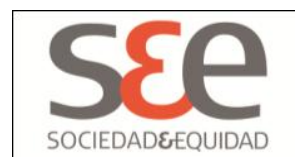

Documental: La CLOC Vía Campesina en Guatemala. Mujeres denuncian ser excluidas y exigen tierra para cultivar. [Documento en línea]. Disponible en:

http: / / www.youtube.com/watch?v=G-fed09Prsl [d23]

Documental: Encuentro Regional de Mujeres de la Vía Campesina Centroamérica. [Documento en línea]. Disponible en:

http: //www.youtube.com/watch?v=D041000yjrk\&feature=related [d24] Documental: Foro Vía Campesina Sudamérica. Rosario. Parte 1, 2 y 3. [documento en línea]. Disponible en: http://www.youtube.com/watch?v=zqQIM6rHusY [d25] Documental: Testimonio del V Congreso CLOC-VC: Alberto Gómez (México). [Documento en línea]. Disponible en: http://www.youtube.com/watch?v=Y-_ZuEl7ZpM [d26] Documental: Testimonio del V Congreso CLOC-VC: Milton Yulán (Ecuador). [Documento en línea]. Disponible en: http://www.youtube.com/watch?v=II3FqYxW5Hk [d27] Documental: Testimonio del V Congreso CLOC-VC: Rafael Alegría (Honduras). [Documento en línea]. Disponible en: http://www.youtube.com/watch?v=E634LhuTTmQ [d28]

Documental: Juventud lucha por el Buen Vivir a través de la Cosmovisión Maya. CLOC Vía Campesina. [Documento en línea]. Disponible en:

http: / / www.youtube.com/watch?v=ulTVOYv2DHA [d29] 